\title{
A new collocation method based on Euler polynomials for solution of generalized pantograph equations
}

\author{
Birol Ibis ${ }^{1}$, Mustafa Bayram ${ }^{2}$ \\ ${ }^{1}$ Department of Basic Sciences, Turkish Air Force Academy, Istanbul,Turkey \\ ${ }^{2}$ Computer Engineering, Faculty of Engineering and Architecture, Istanbul Gelisim University, Istanbul, Turkey
}

Received: 7 December 2016, Accepted: 17 december 2016

Published online: 23 December 2016.

\begin{abstract}
In this paper, a new collocation method based on Euler polynomials is improved for the numerical solution of generalized pantograph equations. This method transforms the generalized pantograph equations into the matrix equation with the help of Euler polynomials and collocation points. This matrix equation corresponds to a system of linear algebraic equations with the unknown Euler coefficients. By solving this system, the unknown Euler coefficients of the solution are found. Some numerical examples are given and comparisons with other methods are made in order to demonstrate the applicability and validity of the proposed method.
\end{abstract}

Keywords: Euler collocation method (ECM), collocation points, Euler polynomial, Pantograph equations.

\section{Introduction}

The pantograph equations are one of the important classes of delay differential equations, which arise in many scientific models such as population studies, controls of mechanical systems, medical biology, electrodynamics and dynamical systems etc., and they also arise in modeling of various phenomena in science and engineering $[1,2,3]$. These equations have been investigated by many authors and both analytical and numerical methods have been developed, some of which are Runge-Kutta and modified Runge-Kutta methods [4,5], differential transform method [6], Taylor collocation method [7], variational iteration method [8,9], homotopy perturbation method [10], $\varepsilon$-Approximate polynomial method [11], shifted Chebyshev polynomial approximation [12] and various collocation methods [13,14,15,16,17,18, 19].

The main idea of the collocation method is to seek the unknown solution function in the form of a linear combination of some basis functions with unknown coefficients. Here, basis functions can be preferred as orthogonal polynomials according to their particular properties, which make them especially ideal for a problem under consideration. In recent years, the various collocation methods have been studied by many authors to obtain solutions of problems arising in different fields of science and engineering [7,12,13,14,15,16,17, 18, 19,20,21,22,23, 24, 25, 26].

In this paper, a new collocation method based on Euler polynomials is proposed to solve the following generalized pantograph equation numerically

$$
y^{(n)}(t)=\sum_{j=0}^{J} \sum_{k=0}^{n-1} p_{j k}(t) y^{(k)}\left(\lambda_{j k} t+\mu_{j k}\right)+g(t), t \geq 0
$$

with the initial conditions

$$
y^{(i)}(0)=c_{i}, \quad i=0,1,2, \ldots, n-1
$$

where $p_{j k}(t)$ and $g(t)$ are given analytical functions, and $\lambda_{j k}, \mu_{j k}, c_{i}$ are appropriate constants. 


\section{Euler polynomials and their some properties}

Euler polynomials and numbers, introduced by Euler in 1740, have special properties and applications in different fields of mathematics like analysis, number theory, differential geometry and algebraic topology. The various properties and relationships involving these polynomials found in many books. The explicit form of well-known Euler polynomials of $\mathrm{n}$-th degree is defined as

$$
E_{n}(t)=\sum_{k=0}^{n}\left(\begin{array}{l}
n \\
k
\end{array}\right) \frac{E_{k}}{2^{k}}\left(t-\frac{1}{2}\right)^{n-k}, n \in N
$$

where $E_{k}$ are Euler numbers. The Euler polynomials have the following known relation for $n \in N^{+}$

$$
E_{n}^{\prime}(t)=n E_{n-1}(t)
$$

The first Euler polynomial is $E_{0}(t)=1$, and the next four are as follows

$$
E_{1}(t)=t-\frac{1}{2}, E_{2}(t)=t^{2}-t, E_{3}(t)=t^{3}-\frac{3}{2} t^{2}+\frac{1}{4}, E_{4}(t)=t^{4}-2 t^{3}+t
$$

If Euler polynomial is presented as a vector in the form $E(t)=\left[\begin{array}{llll}E_{0}(t) & E_{1}(t) & \cdots & E_{N}(t)\end{array}\right]$, then the derivative of the $E(t)$, using (4), can be denoted in the matrix form by

$$
\left[E^{\prime}(t)\right]^{T}=M[E(t)]^{T}
$$

where

$$
\begin{aligned}
& E(t)=\left[\begin{array}{llll}
E_{0}(t) & E_{1}(t) \cdots & E_{N-1}(t) E_{N}(t)
\end{array}\right],
\end{aligned}
$$

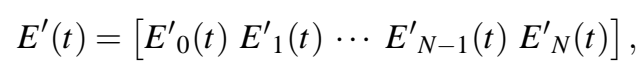

$$
\begin{aligned}
& M=\left[\begin{array}{cccccc}
0 & 0 & \cdots & 0 & 0 & 0 \\
1 & 0 & \cdots & 0 & 0 & 0 \\
0 & 2 & \cdots & 0 & 0 & 0 \\
\vdots & \vdots & \ddots & \vdots & \vdots & \vdots \\
0 & 0 & \cdots & (N-1) & 0 & 0 \\
0 & 0 & \cdots & 0 & N & 0
\end{array}\right]_{(N+1) \times(N+1)}
\end{aligned}
$$

Accordingly, the kth derivative with respect to $t$ of $E(t)$ can be obtained by

$$
\begin{aligned}
{\left[E^{\prime}(t)\right]^{T} } & =M[E(t)]^{T} \Rightarrow E^{\prime}(t)=E(t) M^{T}, \\
E^{\prime \prime}(t) & =E^{\prime}(t) M^{T}=E(t)\left(M^{T}\right)^{2}, \\
E^{\prime \prime \prime}(t) & =E^{\prime}(t)\left(M^{T}\right)^{2}=E(t)\left(M^{T}\right)^{3}, \\
\vdots & \\
E^{(k)}(t) & =E^{(k-1)}(t)\left(M^{T}\right)^{k-1}=E(t)\left(M^{T}\right)^{k},
\end{aligned}
$$

where $\mathrm{M}$ is the Euler operational matrix of derivative.

\section{Euler collocation method (ECM)}

First, we suppose that the solution of the (1) is expressed in the form

$$
y(t)=\sum_{j=0}^{N} a_{j} E_{j}(t),
$$


where $N$ is any chosen positive integer, $a_{j}$ are unknown Euler coefficients, and $E_{j}(t)$ are the Euler polynomials. Then, the approximate solution $y(t)$ given by (7) can be written in matrix form as:

$$
y(t)=E(t) A
$$

where the Euler vector $E(t)$ and the Euler coefficient vector $A$ are given by

$$
\begin{gathered}
E(t)=\left[\begin{array}{llll}
E_{0}(t) & E_{1}(t) & \cdots & E_{N}(t)
\end{array}\right] \\
A^{T}=\left[\begin{array}{llll}
a_{0} & a_{1} & \cdots & a_{N}
\end{array}\right]
\end{gathered}
$$

respectively. With the help of (6), the $k$ th derivative of $y(t)$ can be expressed in the matrix form by

$$
y_{N}^{(k)}(t)=E^{(k)}(t) A
$$

By using relations (6) and (10), we obtain matrix relations

$$
y_{N}^{(k)}(t)=E(t)\left(M^{T}\right)^{k} A .
$$

By substituting (8) and (11) into (1), we get

$$
E(t)\left(M^{T}\right)^{n} A=\sum_{j=0}^{J} \sum_{k=0}^{n-1} p_{j k}(t) E\left(\lambda_{j k} t+\mu_{j k}\right)\left(M^{T}\right)^{k} A+g(t)
$$

where

$$
E\left(\lambda_{j k} t+\mu_{j k}\right)=\left[E_{0}\left(\lambda_{j k} t+\mu_{j k}\right) E_{1}\left(\lambda_{j k} t+\mu_{j k}\right) \cdots E_{N}\left(\lambda_{j k} t+\mu_{j k}\right)\right] .
$$

To obtain the unknown Euler coefficients, the collocation points $t_{i}=i / N, i=0,1,2, \ldots, N$ are put into (12) and the systems of the matrix equations are obtained as

$$
E\left(t_{i}\right)\left(M^{T}\right)^{n} A=\sum_{j=0}^{J} \sum_{k=0}^{n-1} p_{j k}\left(t_{i}\right) E\left(\lambda_{j k} t_{i}+\mu_{j k}\right)\left(M^{T}\right)^{k} A+g\left(t_{i}\right) .
$$

This system can be rescripted as follows

$$
\left\{\tilde{E}\left(M^{T}\right)^{n}-\sum_{j=0}^{J} \sum_{k=0}^{n-1} P_{j k} \tilde{E}_{j k}\left(M^{T}\right)^{k}\right\} A=G
$$

where

$$
\begin{gathered}
\tilde{E}=\left[\begin{array}{cccc}
E_{0}\left(t_{0}\right) & E_{1}\left(t_{0}\right) & \cdots & E_{N}\left(t_{0}\right) \\
E_{0}\left(t_{1}\right) & E_{1}\left(t_{1}\right) & \cdots & E_{N}\left(t_{1}\right) \\
\vdots & \vdots & \ddots & \vdots \\
E_{0}\left(t_{N}\right) & E_{1}\left(t_{N}\right) & \cdots & E_{N}\left(t_{N}\right)
\end{array}\right] \\
\tilde{E}_{i j}=\left[\begin{array}{cccc}
E_{0}\left(\lambda_{j k} t_{0}+\mu_{j k}\right) & E_{1}\left(\lambda_{j k} t_{0}+\mu_{j k}\right) & \cdots & E_{N}\left(\lambda_{j k} t_{0}+\mu_{j k}\right) \\
E_{0}\left(\lambda_{j k} t_{1}+\mu_{j k}\right) & E_{1}\left(\lambda_{j k} t_{1}+\mu_{j k}\right) & \cdots & E_{N}\left(\lambda_{j k} t_{1}+\mu_{j k}\right) \\
\vdots & \vdots & \ddots & \vdots \\
E_{0}\left(\lambda_{j k} t_{N}+\mu_{j k}\right) & E_{1}\left(\lambda_{j k} t_{N}+\mu_{j k}\right) & \cdots & E_{N}\left(\lambda_{j k} t_{N}+\mu_{j k}\right)
\end{array}\right]
\end{gathered}
$$




$$
P_{j k}=\left[\begin{array}{cccc}
p_{j k}\left(t_{0}\right) & p_{j k}\left(t_{0}\right) & \cdots & p_{j k}\left(t_{0}\right) \\
p_{j k}\left(t_{1}\right) & p_{j k}\left(t_{1}\right) & \cdots & p_{j k}\left(t_{1}\right) \\
\vdots & \vdots & \ddots & \vdots \\
p_{j k}\left(t_{N}\right) & p_{j k}\left(t_{N}\right) & \cdots & p_{j k}\left(t_{N}\right)
\end{array}\right], G=\left[\begin{array}{c}
g\left(t_{0}\right) \\
g\left(t_{1}\right) \\
\vdots \\
g\left(t_{N}\right)
\end{array}\right]
$$

Briefly, (14) can be written in the form

$$
W A=G \text { or }[W ; G]
$$

where

$$
W=\left\{\tilde{E}\left(M^{T}\right)^{n}-\sum_{j=0}^{J} \sum_{k=0}^{n-1} P_{j k} \tilde{E}_{j k}\left(M^{T}\right)^{k}\right\}
$$

Thus, (1) is transformed into matrix equation which corresponds to a system of $(\mathrm{N}+1)$ linear algebraic equations with unknown Euler coefficients which can be written in following augmented matrix form

$$
[W ; G]=\left[\begin{array}{ccccc}
w_{00} & w_{01} & \cdots & w_{0 N} & ; g\left(t_{0}\right) \\
w_{10} & w_{11} & \cdots & w_{1 N} & ; g\left(t_{1}\right) \\
\vdots & \vdots & \ddots & \vdots & ; \\
w_{N 0} & w_{N 1} & \cdots & w_{N N} & ; g\left(t_{N}\right)
\end{array}\right]
$$

Next, using (7) and (10) at $t=0$, initial conditions given in (2) can be written in the form of matrix representation as

$$
E(0)\left(M^{t}\right)^{i} A=\left[c_{i}\right], \quad i=0,1,2, \ldots, n-1 .
$$

Thus, the matrix form of (2) is:

$$
U_{i} A=\left[c_{i}\right] \text { or }\left[U_{i} ; c_{i}\right], i=0,1,2, \ldots, N-1
$$

where

$$
U_{i}=E(0)\left(M^{t}\right)^{i}=\left[\begin{array}{llll}
u_{i 0} & u_{i 1} & \ldots & u_{i N}
\end{array}\right], i=0,1,2, \ldots, n-1 .
$$

Finally, by replacing the last $\mathrm{n}$ rows of the augmented matrix (16) by the row matrices (18), we reduce the generalized pantograph equation (1) under conditions (2) to the following linear system of algebraic equations

$$
\tilde{W} A=\tilde{G}
$$

where

$$
\tilde{W}=\left[\begin{array}{cccccc}
w_{01} & w_{02} & \cdots & w_{0 N} & ; & g\left(t_{0}\right) \\
w_{10} & w_{11} & \cdots & w_{1 N} & ; & g\left(t_{0}\right) \\
\vdots & \vdots & \ddots & \vdots & ; & \vdots \\
w_{(N-i) 0} & w_{(N-i) 1} & \cdots & w_{(N-i) N} & g\left(t_{N-i}\right) \\
u_{00} & u_{01} & \cdots & u_{0 N} & ; & c_{0} \\
u_{10} & u_{11} & \cdots & u_{1 N} & ; & c_{1} \\
\vdots & \vdots & \ddots & \vdots & ; & \vdots \\
u_{(n-1) 0} & u_{(n-1) 1} & \cdots & u_{(n-1) N} & ; & c_{n-1}
\end{array}\right]
$$

If $\operatorname{rank} \tilde{W}=\operatorname{rank}[\tilde{W}: \tilde{G}]=N+1$, the linear system (19) has a unique solution and the matrix $A$, which is represented Euler coefficients, is determined by $A=(\tilde{W})^{-1} \tilde{G}$. On the other hand, if $\operatorname{det}(\tilde{W})=0$ and $\operatorname{rank} \tilde{W}=\operatorname{rank}[\tilde{W}: \tilde{G}]<N+1$, then we may obtain the particular solutions. Otherwise, if $\operatorname{rank} \tilde{W} \neq \operatorname{rank}[\tilde{W}: \tilde{G}]$, then there is no solution.

\section{Numerical examples}

In this section, some examples are given to illustrate the applicability, effectiveness, and accuracy of ECM. 
Example 1. Firstly, let us consider the following pantograph equation [14,17]

$$
\left\{\begin{array}{l}
y^{\prime \prime}(t)=\frac{3}{4} y(t)+y\left(\frac{t}{2}\right)-t^{2}+2, t \in[0,1] \\
y(0)=y^{\prime}(0)=0
\end{array}\right.
$$

In this problem, $p_{00}(t)=3 / 4, p_{10}(t)=1$ and $g(t)=-t^{2}+2$. By applying ECM with $N=3$, we may write the approximate solution in the form

$$
y_{3}(t)=\sum_{j=0}^{3} a_{j} E_{j}(t)
$$

where $p_{00}(t)=\frac{3}{4}, p_{10}(t)=1, g(t)=-t^{2}+2$. The collocation points for $\mathrm{N}=3$ is calculated as $\left\{t_{0}=0, t_{1}=1 / 3, t_{2}=2 / 3, t_{3}=1\right\}$.From (14), the fundamental matrix equation of (21) is written as

$$
\left\{\tilde{E}\left(M^{T}\right)^{2}-P_{00} \tilde{E}_{00}-P_{10} \tilde{E}_{10}\right\} A=G
$$

where

$$
\begin{aligned}
& P_{00}=\left[\begin{array}{cccc}
3 / 4 & 0 & 0 & 0 \\
0 & 3 / 4 & 0 & 0 \\
0 & 0 & 3 / 4 & 0 \\
0 & 0 & 0 & 3 / 4
\end{array}\right], P_{10}=\left[\begin{array}{llll}
1 & 0 & 0 & 0 \\
0 & 1 & 0 & 0 \\
0 & 0 & 1 & 0 \\
0 & 0 & 0 & 1
\end{array}\right] \\
& \tilde{E}=\left[\begin{array}{cccc}
1 & -1 / 2 & 0 & 1 / 4 \\
1 & -1 / 6 & -2 / 9 & 13 / 108 \\
1 & 1 / 6 & -2 / 9 & -13 / 108 \\
1 & 1 / 2 & 0 & -1 / 4
\end{array}\right] \\
& \tilde{E}_{10}=\left[\begin{array}{cccc}
1-1 / 2 & 0 & 1 / 4 \\
1 & -1 / 3 & -5 / 36 & 23 / 108 \\
1 & -1 / 6 & -2 / 9 & 13 / 108 \\
1 & 0 & -1 / 4 & 0
\end{array}\right] \text {. }
\end{aligned}
$$

Hence the augmented matrix for (21) is computed as

$$
[W ; G]=\left[\begin{array}{cccccc}
-7 / 4 & 7 / 8 & 2 & -55 / 16 & ; & 2 \\
-7 / 4 & 11 / 24 & 83 / 36 & -563 / 432 & ; 17 / 9 \\
-7 / 4 & 1 / 24 & 43 / 18 & 419 / 432 & ; 14 / 9 \\
-7 / 4 & -3 / 8 & 9 / 4 & 51 / 16 & ;
\end{array}\right]
$$

By using (18), the fundamental matrix forms of initial conditions are computed as

$$
\begin{gathered}
{\left[U_{0} ; c_{0}\right]=\left[\begin{array}{llll}
1 & -\frac{1}{2} & 0 & \frac{1}{4}
\end{array}\right]} \\
{\left[U_{1} ; c_{1}\right]=\left[\begin{array}{llll}
0 & 1 & -1 & 0
\end{array}\right]}
\end{gathered}
$$

Therefore, from (20), the new augmented matrix based on the conditions can be obtained as follows

$$
[\tilde{W} ; \tilde{G}]=\left[\begin{array}{cccccc}
-7 / 4 & 7 / 8 & 2 & -55 / 16 & ; & 2 \\
-7 / 4 & 11 / 24 & 83 / 36 & -563 / 432 & ; 17 / 9 \\
1 & -1 / 2 & 0 & 1 / 4 & ; & 0 \\
0 & 1 & -1 & 0 & ; & 0
\end{array}\right]
$$


Solving this system, the unknown Euler coefficients vector is found as follows.

$$
A=\left[\begin{array}{llll}
\frac{1}{2} & 1 & 1 & 0
\end{array}\right]^{T}
$$

By substituting the determined Euler coefficients into (22), the solution of (21) for $\mathrm{N}=3$ is obtained $y_{3}(t)=t^{2}$ which is the exact solution.

Example 2. Now, let us consider the third order pantograph equation [7,8, 15, 17].

$$
\left\{\begin{array}{l}
y^{\prime}(t)=-y(t)+\mu_{1}(t) y(0.5 t)+\mu_{2}(t) y(0.25 t) \\
y(0)=1
\end{array}\right.
$$

where $\mu_{1}(t)=-e^{-0.5 t} \sin (0.5 t), \mu_{2}(t)=-2 e^{-0.75 t} \cos (0.5 t) \sin (0.25 t)$. The exact solution of (24) is $y(t)=e^{-t}$ cost. In this problem, $p_{00}(t)=-1, p_{10}(t)=\mu_{1}(t), p_{20}(t)=\mu_{2}(t)$ and $g(t)=0$. From (14), the fundamental matrix equation of (24) is written as

$$
\left\{\tilde{E} M^{T}-P_{00} \tilde{E}_{00}-P_{10} \tilde{E}_{10}-P_{20} \tilde{E}_{20}\right\} A=G
$$

By applying the ECM for different values of $\mathrm{N}=3, \mathrm{~N}=5$ and $\mathrm{N}=9$, we obtain the approximate solutions. Fig. 1 shows the absolute error functions $e_{N}(t)=\left|y(t)-y_{N}(t)\right|$. Also, in Table 1, the absolute error functions at the determined points of the given interval are compared with other methods.

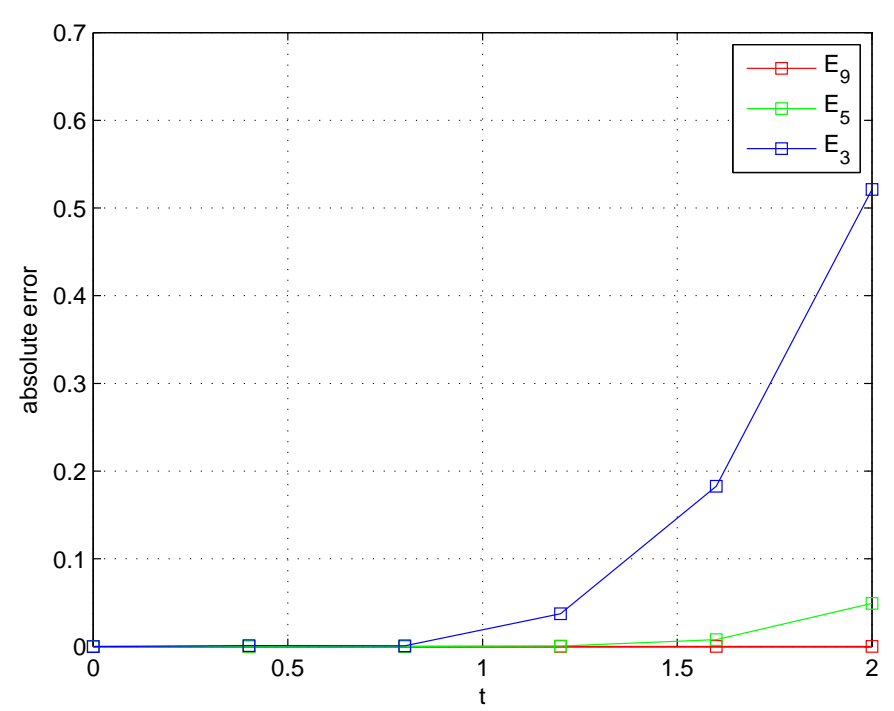

Fig. 1: The absolute error functions $e_{N}(t)$ for $\mathrm{N}=3, \mathrm{~N}=5$ and $\mathrm{N}=9$.

Example 3. Let us consider the following pantograph equation[14, 16, 17, 18].

$$
\left\{\begin{array}{l}
y^{\prime \prime \prime}(t)=-y(t)-y(t-0.3)+e^{-t+0.3}, t \in[0,1] \\
y(0)=1, y^{\prime}(0)=-1, y^{\prime \prime}(0)=1
\end{array}\right.
$$


Table 1: Comparison of the absolute errors corresponding to different methods for (24).

\begin{tabular}{cccccc}
\hline$t_{i}$ & $\begin{array}{c}\text { ECM } \\
\text { N=9 }\end{array}$ & $\begin{array}{c}\text { Ref.[7] } \\
\text { for N=9 }\end{array}$ & Ref.[8] & $\begin{array}{c}\text { Ref.[15] } \\
\text { for N=10 }\end{array}$ & $\begin{array}{c}\text { Ref.[17] } \\
\text { for N=9 }\end{array}$ \\
\hline 0.2 & $8.80 \mathrm{e}-11$ & $1.30 \mathrm{e}-9$ & $5.65 \mathrm{e}-9$ & $5.03 \mathrm{e}-11$ & $1.21 \mathrm{e}-11$ \\
0.4 & $7.89 \mathrm{e}-11$ & $1.43 \mathrm{e}-7$ & $2.95 \mathrm{e}-7$ & $3.98 \mathrm{e}-11$ & $9.68 \mathrm{e}-12$. \\
0.6 & $5.41 \mathrm{e}-11$ & $2.06 \mathrm{e}-6$ & $2.72 \mathrm{e}-6$ & $2.28 \mathrm{e}-11$ & $7.19 \mathrm{e}-12$ \\
0.8 & $8.55 \mathrm{e}-11$ & $1.21 \mathrm{e}-5$ & $1.24 \mathrm{e}-5$ & $1.23 \mathrm{e}-11$ & $6.82 \mathrm{e}-12$ \\
1.0 & $2.23 \mathrm{e}-09$ & $4.00 \mathrm{e}-5$ & $3.80 \mathrm{e}-5$ & $2.25 \mathrm{e}-09$ & $5.62 \mathrm{e}-10$ \\
\hline
\end{tabular}

with the exact solution $y(t)=e^{-t}$.In this problem $p_{00}(t)=-1, p_{10}(t)=-1, g(t)=e^{-t+0.3}$. By using (14), the fundamental matrix equation of (26) is written as

$$
\left\{\tilde{E}\left(M^{T}\right)^{3}-P_{00} \tilde{E}_{00}-P_{10} \tilde{E}_{10}\right\} A=G .
$$

The approximate solution of (26) is obtained for $\mathrm{N}=3,5$ and 9. Fig.2 shows the absolute error functions $E_{N}(t)$ for $\mathrm{N}=3$, $\mathrm{N}=5$, and $\mathrm{N}=9$. Table 2 gives the comparison of the absolute errors obtained by ECM and other methods for $\mathrm{N}=8$.

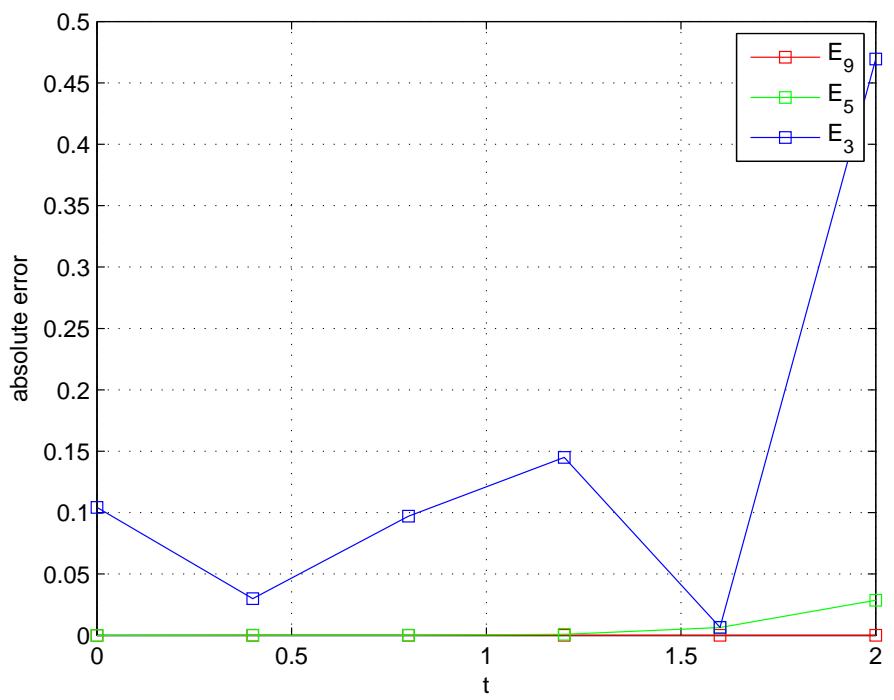

Fig. 2: The absolute error functions $E_{N}(t)$ for $\mathrm{N}=3, \mathrm{~N}=5$ and $\mathrm{N}=9$.

Table 2: Comparison of the absolute errors corresponding to different methods for (26).

\begin{tabular}{cccccc}
\hline \multirow{2}{*}{$t_{i}$} & $\begin{array}{c}\text { ECM } \\
\mathrm{N}=8\end{array}$ & $\begin{array}{c}\text { Ref.[14] } \\
\text { for N=8 }\end{array}$ & $\begin{array}{c}\text { Ref.[16] } \\
\text { for N=8 }\end{array}$ & $\begin{array}{c}\text { Ref.[17] } \\
\text { for N=8 }\end{array}$ & $\begin{array}{c}\text { Ref.[18] } \\
\text { for N=8 }\end{array}$ \\
\hline 0.2 & $6.07 \mathrm{e}-11$ & $6.20 \mathrm{e}-9$ & $3.70 \mathrm{e}-7$ & $1.00 \mathrm{e}-10$ & $5.11 \mathrm{e}-11$ \\
0.4 & $3.06 \mathrm{e}-10$ & $5.76 \mathrm{e}-8$ & $2.38 \mathrm{e}-6$ & $4.00 \mathrm{e}-10$ & $2.50 \mathrm{e}-10$. \\
0.6 & $7.38 \mathrm{e}-10$ & $1.79 \mathrm{e}-7$ & $5.97 \mathrm{e}-6$ & $7.00 \mathrm{e}-10$ & $2.50 \mathrm{e}-10$ \\
0.8 & $9.81 \mathrm{e}-10$ & $3.73 \mathrm{e}-7$ & $3.48 \mathrm{e}-5$ & $8.00 \mathrm{e}-10$ & $7.11 \mathrm{e}-10$ \\
1.0 & $2.62 \mathrm{e}-08$ & $6.36 \mathrm{e}-7$ & $2.03 \mathrm{e}-4$ & $2.69 \mathrm{e}-08$ & $2.68 \mathrm{e}-08$ \\
\hline
\end{tabular}


Example 4. Finaly, let us consider the pantograph equation with variable coefficients.

$$
\left\{\begin{array}{c}
y^{\prime \prime \prime}(t)=\operatorname{ty}\left(\frac{t}{3}-1\right)-t y^{\prime}\left(\frac{t}{4}+1\right)-y(t) \\
\quad-t\left(e^{-\frac{t}{3}+1}+e^{-\frac{t}{4}-1}\right), 0 \leq t \leq 3 \\
y(0)=1, y^{\prime}(0)=-1, y^{\prime \prime}(0)=1
\end{array}\right.
$$

with the exact solution $y(t)=e^{-t}$. Here, $p_{00}(t)=-1, p_{11}(t)=-t, p_{22}(t)=t$ and $g(t)=-t\left(e^{-\frac{t}{3}+1}+e^{-\frac{t}{4}-1}\right)$. From (14), the fundamental matrix equation of the (28) is

$$
\left\{P_{00} \tilde{E}\left(M^{T}\right)^{3}-P_{00} \tilde{E}_{00}-P_{11} \tilde{E}_{11} M^{T}-P_{22} \tilde{E}_{22}\left(M^{T}\right)^{2}\right\} A=G
$$

where $p_{00}(t)=-1, p_{11}(t)=-t, p_{22}(t)=t, g(t)=-t\left(e^{-\frac{t}{3}+1}+e^{-\frac{t}{4}-1}\right)$. By following the present method, we obtain the approximate solutions of (28) for $\mathrm{N}=3,5$ and 9. Fig.3 shows the comparison of the absolute error functions for various values of $\mathrm{N}$.

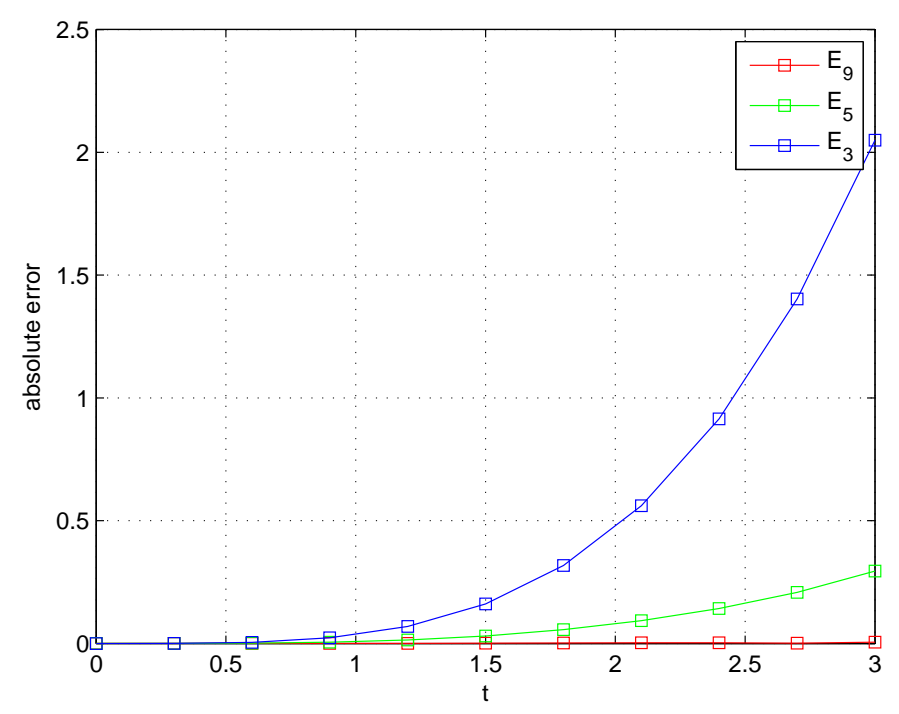

Fig. 3: The absolute error functions $E_{N}(t)$ for $\mathrm{N}=3, \mathrm{~N}=5$ and $\mathrm{N}=9$.

\section{Conclusion}

In this paper, an efficient algorithm, which is named Euler collocation method (ECM), was developed for solving the generalized pantograph equations numerically. This method was illustrated by solving the generalized pantograph equations accurately. By using ECM, these equations were transformed to a linear system of algebraic equations that could be solved easily.

Consequently, the obtained results show that this approach can solve the generalized pantograph equations effectively and this method is consistent with the existing results in the literature. The validity and accuracy of this method is based on the assumption that it converges as the number of collocation points increases. We conclude that the ECM can be considered as an accurate and reliable method for the generalized pantograph equations. 


\section{Competing interests}

The authors declare that they have no competing interests.

\section{Authors' contributions}

All authors have contributed to all parts of the article. All authors read and approved the final manuscript.

\section{References}

[1] J. R. Ockendon and A. B. Tayler. The dynamics of a current collection system for an electric locomotive, Proc. Royal Soc. London A, $332,447-468(1971)$.

[2] W.G. Ajello, H.I. Freedman and J. Wu, A model of stage structured population growth with density depended time delay, SIAMJ.Appl.Math., 52 ,855-869(1992).

[3] Y. Kuang, Delay Differential Equations with Applications in Population Dynamics, Academic, New York, NY, USA, 1993.

[4] D. Li and M. Liu, Runge-Kutta methods for the multi-pantograph delay equation, Appl. Math. Comput.,163(1),383-395(2005).

[5] M. Liu, Z. Yang and Y. Xu, The stability of modified Runge-Kutta methods for the pantograph equation, Math. Comput., 75, 1201-1216(2006).

[6] Y. Keskin, A. Kurnaz, M. Kiris and G. Oturanc, Approximate solutions of generalized pantograph equations by the differential transform method, Int. J. Nonlinear Sci. Numer. Simul., 8, 159-164(2007).

[7] M. Sezer, S.Yalcinbas and N. Sahin, Approximate solution of multi-pantograph equation with variable coefficients, J. Comput. Appl. Math., $214,406-416(2008)$.

[8] Z. Yu, Variational iteration method for solving the multi-pantograph delay equation, Phys.Lett. A, 372, 6475-6479(2008).

[9] A. Saadatmandi and M. Dehghan, Variational iteration method for solving a generalized pantograph equation, Comput.Math.Appl., 58(11-12), 2190-2196(2009).

[10] X. Feng, An analytic study on the multi-pantograph delay equations with variable coefficients, Bull. Math. Soc. Sci. Math. Roumanie, 56(2) , 205-215(2013).

[11] C. Bota and B. Caruntu, $\varepsilon$-Approximate polynomial solutions for the multi-pantograph equation with variable coefficients, Appl. Math. Comput.,219(4), 1785-1792(2012).

[12] S. Sedaghat, Y. Ordokhani and M. Dehghan,Numerical solution of the delay differential equations of pantograph type via Chebyshev polynomials, Commun. Nonlinear Sci., 17(12), 4815-4830(2012).

[13] S. Yuzbasi, An efficient algorithm for solving multipantograph equation systems, Comput. Math. Appl., 64(4),589-603(2012).

[14] S. Yalcinbas, M. Aynigul and M. Sezer, A collocation method using Hermite polynomials for approximate solution of pantograph equations, J. Frankl. Ins., 348(6),1128-1139(2011).

[15] O.R. Isik, Z. Guney and M. Sezer, Bernstein series solutions of pantograph equations using polynomial interpolation, J. Differ. Eq. Appl. ,18(3),357-374(2012).

[16] Y. Ozturk, M. Gulsu, Approximate solution of linear generalized pantograph equations with variable coefficients on ChebyshevGauss grid, J. Adv. Res.Sci. Comput.,4,36-51(2012).

[17] T. Akkaya, S. Yalcinbas and M. Sezer, Numeric solutions for the pantograph type delay differential equation using First Boubaker polynomials, Appl. Math. Comput., 219(17),9484-9492(2013).

[18] E. Tohidi, A.H. Bhrawy and K. Erfani, A collocation method based on Bernoulli operational matrix for numerical solution of generalized pantograph equation , Appl. Math. Model., 37(6),4283-4294(2013).

[19] E.H. Doha, A.H. Bhrawy, D. Baleanu and R.M. Hafez, A new Jacobi rational-Gauss collocation method for numerical solution of generalized pantograph equations, Appl. Numer. Math.,77,43-54(2014).

[20] M. Gulsu, M. Sezer and Z. Guney, Approximate solution of general high-order linear nonhomogeneous difference equations by means of Taylor collocation method, Appl. Math. Comput.,173(2), 683-693(2006).

[21] S. Esmaeili, M. Shamsi and Y. Luchko, Numerical solution of fractional differential equations with a collocation method based on Muntz polynomials,Comput. Math. Appl.,62(3), 918-929(2011).

[22] M. Gulsu, B. Gurbuz, Y. Ozturk and M. Sezer, Laguerre polynomial approach for solving linear delay difference equations, Appl. Math. Comput., 217(15), 6765-6776(2011). 
[23] A.H. Bhrawy and A.S. Alofi, A Jacobi-Gauss collocation method for solving nonlinear Lane-Emden type equations, Commun. Nonlinear Sci., 17(1) , 62-70(2012).

[24] K. Maleknejad, B. Basirat and E. Hashemizadeh, A Bernstein operational matrix approach for solving a system of high order linear Volterra-Fredholm integro-differential equations,Math. Comput. Model., 55(3-4), 1363-1372(2012).

[25] M. M. Khader,N. H. Sweilam and A. M. S. Mahdy, Numerical Study for the Fractional Differential Equations Generated by Optimization Problem Using Chebyshev Collocation Method and FDM, Appl. Math. Inf. Sci.,7(5),2011-2018(2013).

[26] E. Celik, M. Bayram, The numerical solution of physical problems modeled, as a systems of differential-algebraic equations (DAEs), Journal of The Franklin Institute-Engineering And Applied Mathematics, 342(1), 1-6 (2005). 